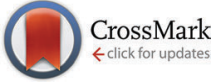

Cite this: Phys. Chem. Chem. Phys., 2017, 19, 7568

Received 27th October 2016 Accepted 14th February 2017 DOI: $10.1039 / c 6 c p 07368 a$ rsc.li/pccp

\section{Two-photon absorption of the spatially confined LiH molecule}

\author{
Justyna Kozłowska, ${ }^{*}$ Marta Chołuj, Robert Zaleśny and Wojciech Bartkowiak* \\ In the present contribution we study the influence of spatial restriction on the two-photon dipole \\ transitions between the $X^{1} \Sigma^{+}$and $A^{1} \Sigma^{+}$states of lithium hydride. The bond-length dependence of the \\ two-photon absorption strength is also analyzed for the first time in the literature. The highly accurate \\ multiconfiguration self-consistent field (MCSCF) method and response theory are used to characterize \\ the electronic structure of the studied molecule. In order to render the effect of orbital compression we \\ apply a two-dimensional harmonic oscillator potential, mimicking the topology of cylindrical confining \\ environments (e.g. carbon nanotubes, quantum wires). Among others, the obtained results provide \\ evidence that at large internuclear distances the TPA response of lithium hydride may be significantly \\ enhanced and this effect is much more pronounced upon embedding of the LiH molecule in an \\ external confining potential. To understand the origin of the observed variation in the two-photon \\ absorption response a two-level approximation is employed.
}

\section{Introduction}

Studies concerning the spatial confinement phenomenon and its influence on the variety of physical and chemical properties of quantum objects have been attracting increasing research attention. This has been triggered by great advances in nanotechnology as well as the rapid development of chemical synthesis methods, particularly in supramolecular chemistry. These factors open up the possibility of constructing molecular systems with entirely new properties, mostly determined by size effects (e.g. endohedral complexes, inclusion compounds or low-dimensional semiconductor structures). ${ }^{1-7}$ A first glimpse of the confinement-induced changes in the chemical and physical properties of atoms or molecules may be caught on a purely theoretical basis through different types of analytical external potentials (e.g. spherical and cylindrical harmonic oscillator potentials, ${ }^{8-14}$ penetrable and impenetrable boxes ${ }^{8,12,15-17}$ ) or by applying a supermolecular approach..$^{8,18-26}$ The analytical based methods allow one to gain an insight into e.g. structural and spectroscopic properties or chemical reactivities of various molecular systems trapped inside confining cavities. For a recent review on the subject, see for example ref. 8 and 27-30.

Another area of research of increasing prominence concerns linear and nonlinear electric properties of spatially restricted atoms, ions and molecules. Basically, it is expected that embedding a quantum system in the confining cages will affect its electronic

Department of Physical and Quantum Chemistry, Wrocław University of Science and Technology, Wybrzeże Wyspiańskiego 27, PL-50370 Wrocław, Poland. E-mail: justyna.kozlowska@pwr.edu.pl,wojciech.bartkowiak@pwr.edu.pl density distribution which, in turn, may be reflected through changes in linear and nonlinear optical (L\&NLO) phenomena. Thus far, numerous theoretical results presented in the literature demonstrate that spatial restriction significantly modifies nonresonant electric dipole properties of atoms and molecules. ${ }^{9-14,17-19,23,24,31,32}$ In particular, it was reported that the values of effective linear polarizability $(\alpha)$ as well as second hyperpolarizability $(\gamma)$ decrease together with the increasing strength of orbital compression..$^{9,10,12,13,17-19,31,33}$ On the other hand, the behavior of dipole moment $(\mu)$ and first hyperpolarizability $(\beta)$ differs depending on the topology of the confining environment and the system under consideration.,12,32,33 It should be underscored that there are only a limited number of theoretical studies concerning the evaluation of the molecular quantities that govern the NLO processes in the resonant regime upon confinement. ${ }^{9}$ This work aims to fill the existing gap. In so doing, in the present contribution the focus is put on the exploration of the effect of spatial confinement on the two-photon absorption (TPA) response of a model molecular system.

TPA, which is a third-order NLO phenomenon, may be described as the electronic excitation of a quantum object induced by the simultaneous absorption of two photons of the same or different energy and, in general, is characterized by several attractive features. Besides the benefits of application of the TPA phenomenon in the field of spectroscopy (it enables the exploration of spectroscopic states which are one-photon forbidden due to symmetry), there are also a number of technological applications of this NLO process. ${ }^{34}$ These include high-resolution fluorescence microscopy, ${ }^{35,36}$ fabrication of 
optoelectronic logical circuits, ${ }^{37,38}$ three-dimensional optical data storage $^{39}$ or nondestructive imaging of biological tissues, ${ }^{35,40}$ just to name a few. As the development of multiphoton based applications relies on the quest for chromophores with large TPA responses, considerable efforts are directed toward the design of appropriate molecular species. ${ }^{34,41,42}$ Although initially the attention was mainly focused on push-pull dipolar molecular structures, ${ }^{42,43}$ over the years it has been shifted to quadrupoles, ${ }^{44,45}$ multichromophoric dendrimeric systems ${ }^{46,47}$ or nanodots. ${ }^{48,49}$ Another promising route to accomplish large TPA responses involves alteration of bond lengths. Particularly, it has been shown that the molecular (hyper)polarizabilities as well as the TPA probability $\left(\delta^{\mathrm{gf}}\right)$ exhibit nonmonotonic changes as a function of the bond-length alternation parameter. ${ }^{50,51}$ Moreover, the results of theoretical and experimental studies clearly indicate that environmental effects, especially solvent polarity, significantly influence the TPA strength of molecular systems. ${ }^{52-54}$

According to the results of some experimental studies, the spatial confinement effect may be considered as another important factor contributing to the changes of TPA strength. ${ }^{55-59}$ For example, it was demonstrated that exposing molecular systems to high pressure leads to the reduction of both one- and twophoton absorption responses. ${ }^{55}$ On the contrary, an enhancement of $\delta^{\text {gf }}$ was reported for different organic molecules confined between the interlayer spaces of clay minerals. ${ }^{5,59}$ Some important conclusions might be also found in recent work concerning the properties of different molecular species enclosed inside metal-organic framework (MOF) materials, which are emerging as unique structures due to their extraordinarily high porosity. Particularly interesting is the observation that systems containing chromophores incorporated into the MOF pores exhibit very strong TPA intensities. ${ }^{57,60}$ Thereby, in addition to the already known potential applications of MOFs (e.g. drug delivery, catalysis or hydrogen storage) they are also considered as an element of new two-photon-pumped microlasers. ${ }^{60}$ In some measure, these findings are in line with those emerging from our recent study performed on the HCCCN molecule embedded in a repulsive potential of cylindrical symmetry. ${ }^{9}$ Based on the conducted analysis it was found that the absolute value of the second-order transition moment $\left(S_{i j}^{\text {gf }}\right)$ increases together with the increasing confinement strength. To the best of our knowledge the study in question provides still the only ab initio results quantifying the influence of spatial confinement on the resonant NLO properties.

The recent experimental studies concerning two-photon absorption properties of molecular species enclosed inside metal-organic framework materials or confined between the interlayer spaces of clay minerals ${ }^{57-60}$ encouraged us to undertake the present investigations. In order to gain a fundamental understanding of various aspects of multiphoton absorption in the presence of spatial confinement, in this article we provide a comprehensive theoretical description of the confinementinduced changes in the two-photon dipole transitions between the $\mathrm{X}^{1} \Sigma^{+}$and $\mathrm{A}^{1} \Sigma^{+}$states of the LiH molecule using high-level electron correlation treatments and response theory. Owing to the simplicity of its electronic structure, lithium hydride is often considered as an ultimate benchmark that allows for a precise assessment of the accuracy and reliability of various theoretical methods. Thus, the number of papers reporting highly accurate reference data for various properties of this molecule is very substantial (see for example ref. 10, 12, 18, 31 and 61-76). Among others, the potential energy curves and spectroscopic properties of many electronic states of $\mathrm{LiH}$ have been already thoroughly investigated..$^{61-65}$ It is also worth noticing that several high quality theoretical studies are available concerning the electronic and vibrational contributions to the dipole moment and (hyper)polarizability of lithium hydride. ${ }^{10,12,18,31,66-76}$ However, with the notable exception of the study devoted to simulations of NLO properties of $\mathrm{LiH}$ using damped cubic response theory within TDDFT, ${ }^{77}$ we are not aware of previous experimental or theoretical studies on the TPA response of the LiH molecule, particularly under usual external conditions. Thus, although the main focus of this work is to get a deeper insight into the influence of orbital compression on the two-photon absorption phenomena, the value of $\delta^{\text {gf }}$ reported here for the unconfined $\mathrm{LiH}$ molecule might be also of significance for subsequent analysis. A further interest of this study is to explore the bond-length dependence of the investigated molecular quantities, for both free and spatially restricted lithium hydride.

\section{Methodology}

The influence of the spatial restriction on the one- and twophoton dipole transitions between the $\mathrm{X}^{1} \Sigma^{+}$and $\mathrm{A}^{1} \Sigma^{+}$states of the LiH molecule was studied by applying a two-dimensional harmonic oscillator (HO) potential. This one-particle confining potential might be expressed as

$$
V_{\mathrm{conf}}=\frac{1}{2} \varphi^{2}\left(x_{i}^{2}+y_{i}^{2}\right)
$$

where $\varphi$ denotes a constant that allows the strength of orbital compression to be controlled. The $\varphi$ values equal to 0.1 and 0.2 were considered in this study. This range roughly corresponds to exchange repulsion energy between linear few-atomic molecules and carbon nanotubes. ${ }^{9,14}$ In all computations the principal axis of the HO potential overlaps with the molecular axis of lithium hydride, assumed to be the $z$-axis. Thus, within the Born-Oppenheimer approximation, adopted in this work, the potential defined by eqn (1) acts only on the electrons of the confined system. Considering the adopted model of spatial restriction the excitation of guest molecules randomly oriented in cylindrical cavities in zeolites by a propagating light beam may constitute a good illustration of the studied phenomenon. Likewise, endohedrally functionalized carbon nanotubes, that can rotate freely in gaseous or liquid medium, can serve as another illustrative example (supposing that the guest molecules are not subjected to rotations). At this stage, it is worth pointing out that different forms of the HO potential proved to be very useful in the description of a variety of physical and chemical environments in condensed-matter physics and 
nanotechnology, ${ }^{8,27,78,79}$ including studies on the electrical properties of confined molecular systems. ${ }^{9-14,19,33}$

In an attempt to assess the effect of spatial restriction on the TPA of the LiH molecule we analyze the values of the secondorder transition moment $\left(S_{i j}^{\text {gf }}\right)$, which constitutes the basic molecular quantity that describes the two-photon absorption process: ${ }^{80}$

$$
S_{i j}^{\mathrm{gf}}=\sum_{k}\left[\frac{\left\langle g\left|\hat{\mu}_{i}\right| k\right\rangle\left\langle k\left|\hat{\mu}_{j}\right| f\right\rangle}{\omega_{k}-\omega}+\frac{\left\langle g\left|\hat{\mu}_{j}\right| k\right\rangle\left\langle k\left|\hat{\mu}_{i}\right| f\right\rangle}{\omega_{k}-\omega}\right] .
$$

In the above equation $|g\rangle$ and $|f\rangle$ correspond to the initial and final state, respectively, while $|k\rangle$ denotes the intermediate state. Labels $i, j$ stand for the Cartesian coordinates and $\left\langle i\left|\hat{\mu}_{i}\right| j\right\rangle$ is the transition moment between states $|i\rangle$ and $|j\rangle$. It is assumed that angular frequencies $(\omega)$ satisfy the resonance condition, i.e. for the one source of photons $2 \omega=\omega_{\mathrm{f}}$. The influence of the external potential on the two-photon absorption probability $\left(\delta^{\mathrm{gf}}\right)$ is also discussed, since this quantity might be related to data extracted from the experimental measurements. ${ }^{81}$ According to the procedure described by Monson and McClain the magnitude of orientationally averaged $\delta^{\text {gf }}$ (hereafter denoted as $\left.\left\langle\delta^{\mathrm{gf}}\right\rangle\right)$ in an isotropic medium might be calculated using the following formula: ${ }^{82}$

$$
\left\langle\delta^{\mathrm{gf}}\right\rangle=\frac{1}{30} \sum_{i j}\left[S_{i i} S_{j j} F+S_{i j} S_{i j} G+S_{i j} S_{j i} H\right],
$$

where the coefficients $F, G$ and $H$ depend on the polarization of the incident light beams; for linearly polarized photons $F=G=$ $H=2$. Note that $\left\langle\delta^{g f}\right\rangle$ is called two-photon absorption strength, by analogy to the oscillator strength $(f)$, which characterizes the one-photon absorption (OPA) process. Both $S_{i j}^{\mathrm{gf}}$ and $\left\langle\delta^{\mathrm{gf}}\right\rangle$ are calculated for the transition to the lowest-lying singlet electronic excited state of lithium hydride, for which a significant intramolecular charge transfer (CT) occurs. In such case the diagonal component of the two-photon transition moment along the symmetry axis (here $S_{z z}^{\mathrm{gf}}$ ) is by far the largest and consequently contributes the most to $\left\langle\delta^{\text {gf }}\right\rangle$. Therefore, although in this article we determine all tensor elements and averaged properties, particular attention will be put on the confinementinduced changes in the values of $S_{z z}^{g f}$.

Under the assumption that the presence of the CT state dominates the response of molecular systems to the external electric field it is possible to reduce the expressions defining the second-order transition moment (eqn (2)) within the wellknown two-level approximation: ${ }^{34,42,83,84}$

$$
S_{z z}^{\mathrm{gf}, \mathrm{TLM}}=4 \frac{\left\langle g\left|\hat{\mu}_{z}\right| f\right\rangle\left(\left\langle f\left|\hat{\mu}_{z}\right| f\right\rangle-\left\langle g\left|\hat{\mu}_{z}\right| g\right\rangle\right)}{\omega_{\mathrm{f}}} .
$$

The importance of the two-level model (TLM) in the field of molecular nonlinear optics stems from the fact that it allows one to define the response of a system in terms of simple spectroscopic parameters, like excitation energy $\left(\omega_{\mathrm{f}}\right)$, transition moment $\left(\left\langle g\left|\hat{\mu}_{z}\right| f\right\rangle\right)$ and change of polarity between ground and low-lying excited charge transfer states $\left(\Delta \mu_{z}=\left\langle f\left|\hat{\mu}_{z}\right| f\right\rangle-\right.$ $\left.\left\langle g\left|\hat{\mu}_{z}\right| g\right\rangle\right)$. By using this relationship it becomes possible to establish how changes in the above mentioned factors, occurring due to the presence of an external confining potential, affect the second-order transition moment value.

In order to characterize the electronic structure of lithium hydride in the $\mathrm{X}^{1} \Sigma^{+}$and $\mathrm{A}^{1} \Sigma^{+}$states the multiconfiguration self-consistent field (MCSCF) method together with response function formalism was used, as implemented in the Dalton package. ${ }^{85}$ Specifically, the MCSCF method was applied for the ground state wave function, whereas the one- and two-photon dipole transition properties were obtained from the multiconfiguration linear and quadratic response functions (MCLR and MCQR). ${ }^{86-89}$ The computations were performed in $C_{2 \mathrm{v}}$ symmetry, using the ANO-L basis set. ${ }^{90}$ All electrons were correlated and all orbitals included in the active space during the MCSCF calculations. It should be noted that the dependence of the analyzed quantities on the internuclear distance $(R)$ was also investigated. Therefore, the one- and two-photon transition dipole moments and excitation energy values, as well as the dipole moment difference between the excited and the ground electronic state, were computed as a function of $R$, both under vacuum and in the presence of HO potential. Such analyses were carried out for the internuclear distances between 1.3 and 20 a.u. It should be noted that although the sign of $S_{z z}^{\mathrm{gf}}$ is undetermined, we checked phases of wave functions and response vectors at each distance to obtain a smooth curve presented in Fig. 1. The same applies to Fig. 2.

\section{Results and discussion}

We shall start the discussion with the analysis of data obtained for the $\mathrm{LiH}$ molecule at the experimental equilibrium distance $\left(R_{\mathrm{e}}=3.015\right.$ a.u.), which are presented in Table 1 . The values of the diagonal component of the second-order transition moment and TPA probability computed at this particular bond length for isolated lithium hydride are equal to 103.9 a.u. and 3760 a.u., respectively. However, the external confining potential causes a substantial drop of $S_{z z}$ and $\left\langle\delta^{g f}\right\rangle$. As one can notice, although the values of the second-order transition moment calculated using the two-level model $\left(S_{z z}^{\text {gf,TLM }}\right)$ noticeably underestimate those determined within the response function formalism $\left(S_{z z}^{\mathrm{gf}}\right)$, both methods predict the same nature of changes in the analyzed quantity upon confinement. Therefore, the two-level approximation seems to be an adequate approach to explain the behavior of $S_{z z}^{\mathrm{gf}}$. From Table 1 it is evident that the reduction of TPA response of spatially limited lithium hydride is caused by two factors: the decrease of the one-photon transition dipole moment value and the hypsochromic shift of the excitation energy between $\mathrm{X}^{1} \Sigma^{+}$and $\mathrm{A}^{1} \Sigma^{+}$states. The latter result closely follows intuitive expectations, as it is well established that the presence of analytical potentials representing the so called "pure" spatial confinement (i.e. no attractive interactions between the confined molecule and its environment) would cause an increase of the gap between frontier orbitals relative to that of unconfined atoms or molecules. ${ }^{16,19,91}$ 

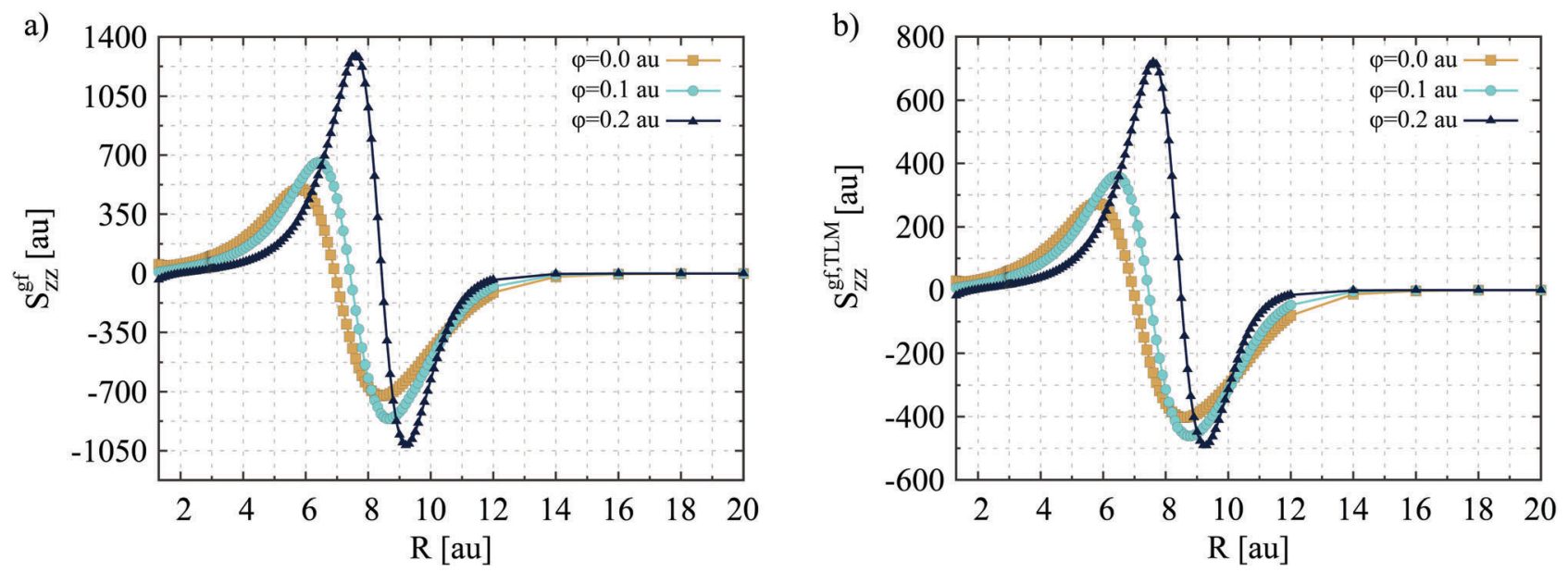

Fig. 1 The second-order transition moment function of the free and spatially confined LiH molecule obtained using the multiconfiguration quadratic response functions (a) and two-level approximation (b). Calculations were performed using the ANO-L basis set.
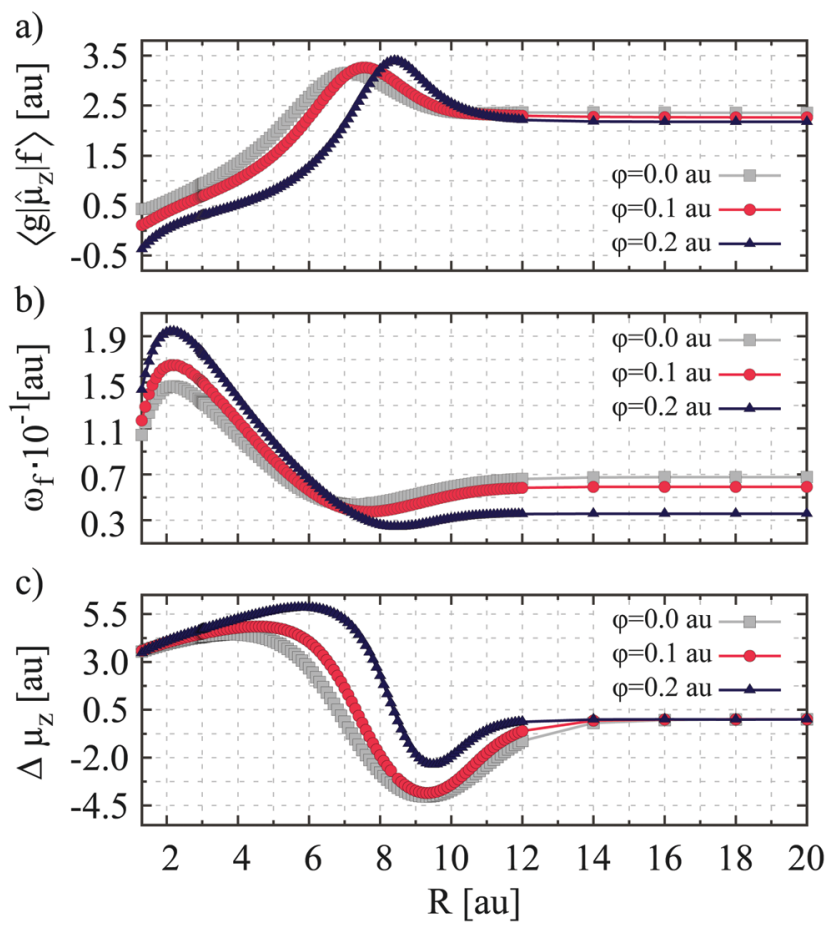

Fig. 2 Transition dipole moment (a), excitation energy (b) and change of polarity (c) between ground $\left(\mathrm{X}^{1} \Sigma^{+}\right)$and low-lying excited charge transfer $\left(\mathrm{A}^{1} \Sigma^{+}\right)$states of the free and spatially confined $\mathrm{LiH}$ molecule, obtained as a function of the internuclear distance. Calculations were performed using the MCSCF wave function and the ANO-L basis set.

The dependence of the diagonal component of the secondorder transition moment of $\mathrm{LiH}$ on the internuclear distance, evaluated using the multiconfiguration quadratic response functions and two-level approximation, is depicted in Fig. 1. At this stage it should be mentioned that the $R$-dependence brings forth important features of the static electric properties $(\mu, \alpha, \beta$ and $\gamma)$ of molecular systems, as it has been already disclosed in many valuable scientific works. ${ }^{10,92-108}$ Although it
Table 1 The values of the diagonal component of the second-order transition moment $\left(S_{z Z}^{g f}, S_{z Z}^{g f, T L M}\right)$ and TPA strength $\left(\left\langle\delta^{g f}\right\rangle\right)$, as well as OPA spectroscopic parameters $\left(\omega_{\mathrm{f}},\left\langle g\left|\hat{\mu}_{z}\right| f\right\rangle, \Delta \mu_{z}\right)$ computed for the free and spatially confined $\mathrm{LiH}$ molecule at the experimental equilibrium distance (3.015 a.u.). Symbols $|g\rangle$ and $|f\rangle$ correspond to $X^{1} \Sigma^{+}$and $A^{1} \Sigma^{+}$states, respectively. Calculations were performed using the MCSCF wave function and the ANO-L basis set. All values are given in a.u.

\begin{tabular}{lrlrlll}
\hline$\varphi$ & \multicolumn{1}{c}{$S_{z z}^{\mathrm{gf}}$} & $S_{z z}^{\mathrm{gf}, \mathrm{TLM}}$ & \multicolumn{1}{c}{$\left\langle\delta^{\mathrm{gf}}\right\rangle$} & \multicolumn{1}{l}{$\omega_{\mathrm{f}}$} & $\left\langle g\left|\hat{\mu}_{z}\right| f\right\rangle$ & $\Delta \mu_{z}$ \\
\hline 0.0 & 103.9 & 60.4 & 3760 & $1.3 \times 10^{-1}$ & $9.3 \times 10^{-1}$ & 4.3 \\
0.1 & 69.7 & 41.9 & 1360 & $1.5 \times 10^{-1}$ & $7.0 \times 10^{-1}$ & 4.5 \\
0.2 & 24.9 & 16.4 & 178 & $1.8 \times 10^{-1}$ & $3.1 \times 10^{-1}$ & 4.7
\end{tabular}

is difficult to draw one general conclusion emerging from these studies, there are several important observations worth underscoring. Among others, the sign inversion of the dipole moment with the change in the internuclear separation is characteristic for various molecular systems (e.g. $\mathrm{AlCl}, \mathrm{AlF}$, $\mathrm{AlH}, \mathrm{BCl}, \mathrm{BF}, \mathrm{CO}, \mathrm{CS}, \mathrm{HBr}, \mathrm{HCl}, \mathrm{HF}, \mathrm{MgHe}$, NaLi, SiO, SiS, YBr). ${ }^{97-100}$ Such observation is of relevance as it reflects the process of electron charge transfer inside the molecule. Moreover, on the basis of extensive theoretical studies Maroulis and co-workers have found that the variation of bond length results in substantial changes of (hyper)polarizability, which are quite distinct for different molecular systems. ${ }^{101-104}$ A thorough consideration of the connection of polarizability and hyperpolarizability derivatives to Raman and hyper-Raman spectra was also reported by Quinet and Champagne. ${ }^{109}$ From the theoretical point of view an important finding concerns also the fact that changes in the intermolecular distance may have a substantial influence on the electron correlation contribution to the studied electric properties. ${ }^{102,104}$

Quite recently, Lo and Klobukowski discussed the electronic structure as well as the response of $\mu$ and $\alpha$ of lithium hydride to the confining potential and also the dependence of the computed quantities on the internuclear distance. ${ }^{10}$ As it was found by the authors, the changes of $\mu$ and $\alpha$ as a function of bond length are substantial and slightly dependent on the 
external potential. The curves displayed in Fig. 1(a) clearly demonstrate that the value of the second-order transition moment of lithium hydride varies largely with the internuclear separation as well. For the sake of discussion performed herein, it should be noted that only the absolute values of $S_{z z}^{\mathrm{gf}}$ are of significance for the magnitude of the TPA response. As one can notice the $S_{z z}^{\mathrm{gf}}$ function exhibits a nonmonotonic behaviour even for the unconfined lithium hydride. Particularly, for $R<R_{\mathrm{e}}$ the values of $S_{z z}^{\mathrm{gf}}$ computed for the free $\mathrm{LiH}$ molecule are smaller than those determined at the experimental equilibrium distance. However, stretching the LiH bond length leads to an increase in the magnitude of the second-order transition moment and the $S_{z z}^{\mathrm{gf}}(R)$ function exhibits two extrema equal to 499 and -723 a.u. at $R=5.8$ a.u. and $R=8.5$ a.u., respectively. Note that in the intermediate internuclear separation range the inversion of the $S_{z z}^{g f}$ sign occurs, while for large $R(R>16$ a.u.) its value converges to zero.

Turning the attention to the results obtained for the spatially limited LiH molecule several interesting conclusions can be also drawn. As it turns out, upon embedding in the harmonic potential the second-order transition moment of $\mathrm{LiH}$ follows, in general, the same patterns of changes as presented by the unconfined molecule. Yet, the internuclear distances at which $S_{z z}^{\mathrm{gf}}$ reaches its maxima (extrema) are shifted toward larger values of $R$. Likewise, the bond lengths for which inversion of the second-order transition moment sign is observed (so called "crossing point") are also noticeably larger. Obviously, the shifts in the $S_{z z}^{\mathrm{gf}}(R)$ function can be considered as a natural consequence of the fact that the employed confining potential causes an increase of the energy gap between the $\mathrm{X}^{1} \Sigma^{+}$and $\mathrm{A}^{1} \Sigma^{+}$states of LiH. Moreover, it follows from Fig. 1 that the influence of spatial restriction on the $S_{z z}^{\mathrm{gf}}$ value is much more pronounced far from the equilibrium bond length of lithium hydride. In contrast to what was observed when $R=R_{\mathrm{e}}$, at larger internuclear distances the presence of an external potential results in a significant enhancement of the second-order transition moment with respect to the value obtained for the unconfined $\mathrm{LiH}$ molecule. It is notable that there is a three-fold increase of the maximum values of $S_{z z}^{\mathrm{gf}}$ due to confinement. The above observations can be easily understood by the analysis of key parameters for the maximum, crossing point and minimum on the $S_{z z}^{\mathrm{gf}}$ curve (for all confinement strengths), which are assembled in Table 2. In particular, for the internuclear distances under consideration, the excitation energy decreases by an order of magnitude, while $\left\langle g\left|\hat{\mu}_{z}\right| f\right\rangle$ significantly increases, with respect to the values obtained at the experimental equilibrium distance ( $c f$. Table 1). Moreover, for both free and spatially confined LiH, the crossing points are characterized by smaller $\omega_{\mathrm{f}}$ and $\Delta \mu$ values and larger OPA transition moments when compared to the data obtained for $S_{z z}^{\max }$ and $S_{z z}^{\min }$. According to the TLM, changes in the above mentioned spectroscopic parameters, and their mutual correlation, have a decisive impact on the second-order transition moment values (see the discussion below).

As it follows from the data depicted in Fig. 1(b) the estimated $S_{z z}^{\text {gf,TLM }}$ values reproduce reasonably well those computed using the MCQR approach. Thus, the two-level approximation is
Table 2 The values of the selected OPA spectroscopic parameters computed for the $\mathrm{LiH}$ molecule at bond lengths corresponding to the maximum $\left(S_{z z}^{\max }\right)$, minimum $\left(S_{z z}^{\min }\right)$ and crossing point $\left(S_{z z}^{c p}\right)$ on the secondorder transition moment curve (for all confinement strengths). The term "crossing point" refers to the internuclear distance at which the inversion of the $S_{z z}^{g f}$ sign occurs. Symbols $|g\rangle$ and $|f\rangle$ correspond to $X^{1} \Sigma^{+}$and $A^{1} \Sigma^{+}$ states, respectively. Calculations were performed using the MCSCF wave function and the ANO-L basis set. All values are given in a.u.

\begin{tabular}{llll}
\hline & $S_{z z}^{\max }$ & $S_{z z}^{\mathrm{cp}}$ & $S_{z z}^{\min }$ \\
\hline$\varphi=0.0$ & & & \\
$R$ & 5.80 & 7.00 & 8.50 \\
$\omega_{\mathrm{f}}$ & $5.7 \times 10^{-2}$ & $4.4 \times 10^{-2}$ & $4.9 \times 10^{-2}$ \\
$\left\langle g\left|\hat{\mu}_{z}\right| g\right\rangle$ & -2.9 & -1.8 & -0.5 \\
$\left\langle f\left|\hat{\mu}_{z}\right| f\right\rangle$ & 0.2 & -2.0 & -4.1 \\
$\Delta \mu_{\mathrm{z}}$ & 3.1 & -0.2 & -3.6 \\
$\left\langle g\left|\hat{\mu}_{z}\right| f\right\rangle$ & 2.5 & 3.2 & 2.8 \\
& & & \\
$\varphi=0.1$ & & & \\
$R$ & 6.40 & 3.50 & 8.80 \\
$\omega_{\mathrm{f}}$ & $4.8 \times 10^{-2}$ & -2.0 & $4.3 \times 10^{-2}$ \\
$\left\langle g\left|\hat{\mu}_{z}\right| g\right\rangle$ & -3.3 & -2.2 & -0.6 \\
$\left\langle f\left|\hat{\mu}_{z}\right| f\right\rangle$ & 0.03 & -0.2 & -4.1 \\
$\Delta \mu_{\mathrm{z}}$ & 3.33 & 3.3 & -3.5 \\
$\left\langle g\left|\hat{\mu}_{\mathrm{z}}\right| f\right\rangle$ & 2.6 & & 2.8 \\
$\varphi=0.2$ & & & \\
$R$ & & 8.50 & 9.20 \\
$\omega_{\mathrm{f}}$ & 7.60 & $2.0 \times 10^{-2}$ & $3.0 \times 10^{-2}$ \\
$\left\langle g\left|\hat{\mu}_{z}\right| g\right\rangle$ & $3.0 \times 10^{-2}$ & -2.1 & -0.9 \\
$\left\langle f\left|\hat{\mu}_{z}\right| f\right\rangle$ & -3.9 & -2.2 & -3.1 \\
$\Delta \mu_{\mathrm{z}}$ & -0.1 & -0.1 & 2.2 \\
$\left\langle g\left|\hat{\mu}_{z}\right| f\right\rangle$ & 3.8 & 3.4 & 3.0 \\
& 2.8 & &
\end{tabular}

sufficient to qualitatively explain the changes in the secondorder transition moment as a function of internuclear separation. The dependence of the spectroscopic parameters contributing to $S_{z z}^{\text {gf,TLM }}$ on the bond length is illustrated in Fig. 2. A close inspection of the presented plots allows one to conclude that the change in $S_{z z}^{\mathrm{gf}}$ is mostly governed by the variation of $\Delta \mu_{z}$. Of particular importance are changes occurring for the excited state dipole moment. In this case a maximum and a minimum of the $\mu_{z}(R)$ function appear at internuclear distances close to those for which the peaks of $S_{z z}^{\mathrm{gf}}$ functions are also located. On the other hand, in the ground electronic state of LiH the dipole moment reaches its maximum at bond length, where the potential energy curve crosses the $\mathrm{Li}^{+} \mathrm{H}^{-}$ionic potential curve, ${ }^{10}$ and yields zero values at larger internuclear distances. The enhancement of the second-order transition moment value at $R>R_{\mathrm{e}}$ is also due to the decrease of the excitation energy value, accompanied by an increase of the one photon transition dipole moment. Nevertheless, these two quantities have considerably less impact on the nature of $S_{z z}^{\mathrm{gf}}$ changes in the function of internuclear separation. Noteworthy, this observation applies to both free and spatially limited lithium hydride.

In Fig. 3, the variation of the one- and two-photon absorption strength of $\mathrm{LiH}$ with the internuclear separation is presented. Unsurprisingly, the $R$-dependence of $\left\langle\delta^{\text {gf }}\right\rangle$ bears a strong resemblance to that of the diagonal component of the secondorder transition moment of $\mathrm{LiH}$. The total effect is even more pronounced as the maximum value of TPA strength under double perturbation, that is the confining potential with $\varphi=0.2$ a.u. and 
a)
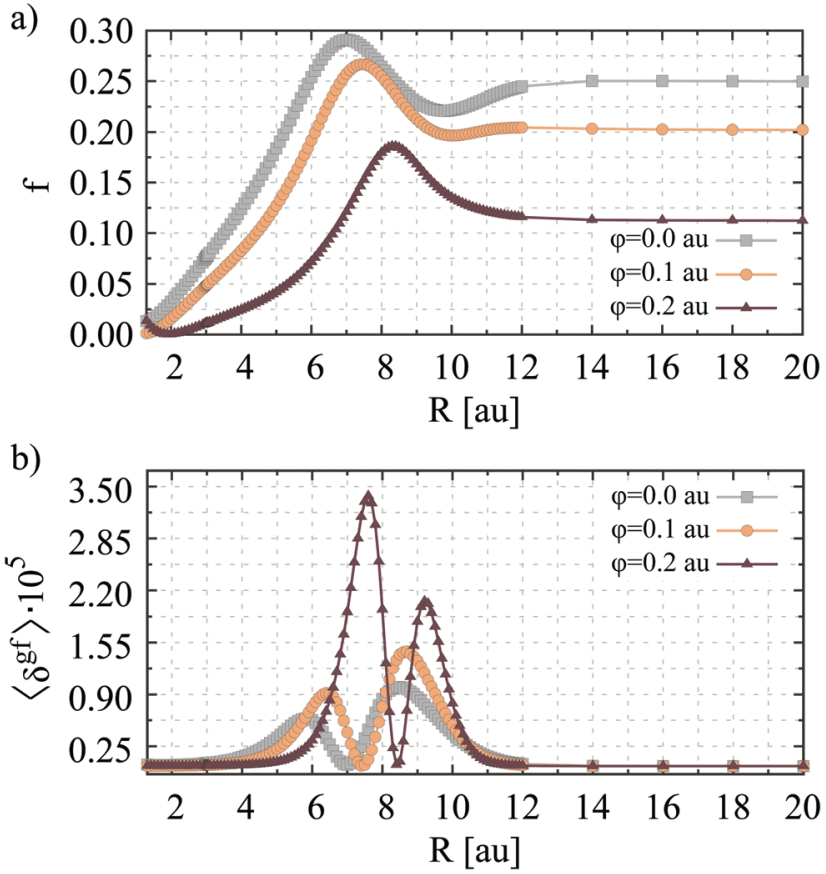

Fig. 3 One- (a) and two-photon (b) absorption strength function of the free and spatially confined $\mathrm{LiH}$ molecule. Calculations were performed using the MCSCF wave function and the ANO-L basis set.

the bond stretched to 7.6 a.u., is almost ninety times larger than $\left\langle\delta^{\mathrm{gf}}\right\rangle$ computed for unconfined $\mathrm{LiH}$ at the experimental equilibrium distance. However, one may find fundamental differences between the changes in the one- and two-photon absorption strength occurring due to the variation in the internuclear separation in the presence of confining HO potential ( $c f$. Fig. 3(a)). Although the $R$-dependence of oscillator strength $(f)$ demonstrates nonmonotonic behavior, for internuclear distances larger than $R_{\mathrm{e}}$ the value of $f$ is always greater than the one computed at the experimental bond distance. This applies to the result obtained under vacuum as well as upon embedding $\mathrm{LiH}$ in $\mathrm{HO}$ potential. In contrast to $\left\langle\delta^{\mathrm{gf}}\right\rangle$ the spatial confinement diminishes the OPA strength virtually in the whole range of $R$. The present findings remain in agreement with the observations made in a theoretical study concerning the absorption spectra of the $p$-nitroaniline (pNA) molecule embedded in different confining cages. ${ }^{22}$ In particular, it was demonstrated that under the influence of chemical pressure, imposed by the helium tube, the absorption maximum of pNA is shifted to larger wavelengths, losing some of its intensity.

\section{Conclusions}

The present contribution provides a theoretical description of the two-photon absorption response of the $\mathrm{LiH}$ molecule embedded in a two-dimensional harmonic oscillator potential, expected to capture the exchange repulsion within the confining environments of cylindrical symmetry. Comprehensive analyses were conducted for the second-order transition moment and TPA probability values evaluated using the multiconfiguration self-consistent field method together with response function formalism. An important aspect of this work was also to explore the bond-length dependence of the investigated molecular quantities, for both free and spatially restricted lithium hydride.

The results of the performed calculations indicate a significant reduction of the two-photon absorption response of lithium hydride at its experimental equilibrium bond length upon confinement. On the other hand, large and nonmonotonic changes of the second-order transition moment value, and consequently TPA strength, were observed due to the variation in the internuclear separation. As it has been found that at distances larger than the equilibrium bond length a substantial enhancement of $S_{z z}^{\mathrm{gf}}$ and $\left\langle\delta^{\mathrm{gf}}\right\rangle$ might occur. Moreover, it has been disclosed that the importance of the orbital compression effect increases in the case of highly distorted geometries of lithium hydride. According to the obtained results under double perturbation, i.e. when the bond length of the $\mathrm{LiH}$ molecule embedded in an external potential is strongly stretched, the TPA strength could increase by several orders of magnitude. Analysis of the results in terms of the two-level model leads to the conclusion that the observed changes in the TPA response are mostly governed by the variation in the difference between the ground- and excited electronic state dipole moment of $\mathrm{LiH}$.

Summing up, the obtained results provide evidence that the "pure" spatial confinement effect might have a significant influence on the magnitude of the two-photon absorption response of molecular systems. According to our knowledge, some of the studied topics, including comprehensive analysis related to bond-length dependence of the investigated molecular quantities, have never been considered in the literature before. Moreover, it should not be overlooked that the highly accurate $a b$ initio values of the second-order transition moment and TPA probability of the unconfined $\mathrm{LiH}$ molecule were reported herein for the first time in the literature.

\section{Acknowledgements}

This work was supported by NCN grant DEC-2013/09/N/ST4/ 00325 and a statutory activity subsidy from the Polish Ministry of Science and Higher Education for the Faculty of Chemistry of Wrocław University of Technology. R. Z. is grateful for the support from the National Science Centre, Poland (Grant No. 2015/19/B/ST4/01881). The authors also acknowledge the Wroclaw Centre for Networking and Supercomputing for the generous allotment of computer time. This research was supported in part by PL-Grid Infrastructure. The authors are grateful to J. Zaremba for his insightful comments on the manuscript.

\section{References}

1 D. L. Caulder and K. N. Raymond, Acc. Chem. Res., 1999, 32, 975-982.

2 F. Hof, S. L. Craig, C. Nuckolls and J. Rebek, Angew. Chem., 2002, 41, 1488-1508. 
3 S. R. Seidel and P. J. Stang, Acc. Chem. Res., 2002, 35, 972-983.

4 Y. Tao, H. Kanoh, L. Abrams and K. Kaneko, Chem. Rev., 2006, 106, 896-910.

5 J. X. Jiang, J. H. Yu and A. Corma, Angew. Chem., 2010, 49, 3120-3145.

6 V. Biju, T. Itoh, A. Anas, A. Sujit and M. Ishikawa, Anal. Bioanal. Chem., 2008, 391, 2469-2495.

7 S. Y. Lim, W. Shen and Z. Gao, Chem. Soc. Rev., 2015, 44, 362-381.

8 Advances in Quantum Chemistry: Theory of Confined Quantum Systems, ed. J. R. Sabin, E. Brändas and S. A. Cruz, Academic Press, Waltham, MA, 2009, vol. 57-58.

9 R. Zaleśny, R. W. Góra, J. Kozłowska, J. M. Luis, H. Ågren and W. Bartkowiak, J. Chem. Theory Comput., 2013, 9, 3463-3472.

10 J. M. H. Lo and M. Klobukowski, Chem. Phys., 2006, 328, 132.

11 S. A. Ndengué, O. Motapon, R. L. Melingui Melono and A. J. Etindele, J. Phys. B: At., Mol. Opt. Phys., 2014, 47, 015002.

12 R. W. Góra, R. Zaleśny, J. Kozłowska, P. Naciażek, A. Roztoczyńska, K. Strasburger and W. Bartkowiak, J. Chem. Phys., 2012, 137, 094307.

13 J. Kozłowska and W. Bartkowiak, Chem. Phys., 2014, 441, 83-92.

14 R. Zaleśny, R. W. Góra, J. M. Luis and W. Bartkowiak, Phys. Chem. Chem. Phys., 2015, 17, 21782-21786.

15 R. Colin-Rodriguez and S. A. Cruz, J. Phys. B: At., Mol. Opt. Phys., 2010, 43, 235102.

16 A. Borgoo, D. J. Tozer, P. Geerlings and F. De Proft, Phys. Chem. Chem. Phys., 2008, 10, 1406-1410.

17 K. Strasburger and P. Naciażek, J. Phys. B: At., Mol. Opt. Phys., 2014, 47, 025002.

18 A. Kaczmarek and W. Bartkowiak, Phys. Chem. Chem. Phys., 2009, 11, 2885.

19 J. Kozłowska, R. Zaleśny and W. Bartkowiak, Chem. Phys., 2014, 428, 19-28.

20 C. N. Ramachandran, D. De Fazio, N. Sathyamurthy and V. Aquilanti, Chem. Phys. Lett., 2009, 473, 146.

21 N. A. Besley and A. Noble, J. Chem. Phys., 2008, 128, 101102. 22 A. Kaczmarek-Kedziera, J. Phys. Chem. A, 2011, 115, 5210.

23 F. Ma, Z. Zhou and Y. Liu, ChemPhysChem, 2012, 13, 1307.

24 B. Ensing, F. Costanzo and P. L. Silvestrelli, J. Phys. Chem. A, 2012, 116, 12184.

25 A. Roztoczyńska, J. Kozłowska, P. Lipkowski and W. Bartkowiak, Phys. Chem. Chem. Phys., 2016, 18, 2417-2427.

26 M. Pagliai, G. Cardini and R. Cammi, J. Phys. Chem. A, 2014, 118, 5098-5111.

27 Electronic Structure of Quantum Confined Atoms and Molecules, ed. E. K. D. Sen, Springer, 2014.

28 V. Schettino and R. Bini, Chem. Soc. Rev., 2007, 36, 869.

29 K. E. Gubbins, Y.-C. Liu, J. D. Moorea and J. C. Palmera, Phys. Chem. Chem. Phys., 2011, 13, 58-85.

30 Z. V. Todres, Free Preview Organic Chemistry in Confining Media, Springer, New York, 2013.

31 W. Bartkowiak and K. Strasburger, J. Mol. Struct.: THEOCHEM, 2010, 960, 93.

32 S. A. Cruz and J. Soullard, Chem. Phys. Lett., 2004, 391, 138.
33 J. Kozłowska, A. Roztoczyńska and W. Bartkowiak, Chem. Phys., 2015, 456, 98-105.

34 M. Pawlicki, H. A Collins, R. G. Denning and H. L. Anderson, Angew. Chem., Int. Ed., 2009, 48, 3244-3266.

35 F. Helmchen and W. Denk, Nat. Methods, 2005, 2, 932-940.

36 G. C. Cianci, J. R. Wu and K. M. Berland, Microsc. Res. Tech., 2004, 64, 135-141.

37 C. R. Mendonca, D. S. Correa, F. Marlow, T. Voss, P. Tayalia and E. Mazur, Appl. Phys. Lett., 2009, 95, 113309.

38 M. P. Joshi, H. E. Pudavar, J. Swiatkiewicz, P. N. Prasad and B. A. Reianhardt, Appl. Phys. Lett., 1999, 74, 170-172.

39 K. D. Belfield and K. J. Schafer, Chem. Mater., 2002, 4, 3656-3662.

40 K. Konig, J. Microsc., 2000, 200, 83-104.

41 M. Albota, D. Beljonne, J. L. Brédas, J. E. Ehrlich, J. Y. Fu, A. A. Heikal, S. E. Hess, T. Kogej, M. D. Levin, S. R. Marder, D. McCord-Maughon, J. W. Perry, H. Röckel, M. Rumi, G. Subramaniam, W. W. Webb, X. L. Wu and C. Xu, Science, 1998, 281, 1653-1656.

42 F. Terenziani, C. Katan, E. Badaeva, S. Tretiak and M. Blanchard-Desce, Adv. Mater., 2008, 20, 4641-4678.

43 B. A. Reinhardt, L. L. Brott, S. J. Clarson, A. G. Dillard, J. C. Bhatt, R. Kannan, L. X. Yuan, G. S. He and P. N. Prasad, Chem. Mater., 1998, 10, 1863-1874.

44 M. Charlot, N. Izard, O. Mongin, D. Riehl and M. BlanchardDesce, Chem. Phys. Lett., 2006, 417, 297-302.

45 G. P. Bartholomew, M. Rumi, S. J. K. Pond, J. W. Perry, S. Tretiak and G. C. Bazan, J. Am. Chem. Soc., 2004, 126, 11529-11542.

46 R. L. Roberts, T. Schwich, T. Ch. Corkery, M. P. Cifuentes, K. A. Green, J. D. Farmer, P. J. Low, T. B. Marder, M. Samoć and M. G. Humphrey, Adv. Mater., 2009, 21, 2318-2322.

47 K. A. Green, M. P. Cifuentes, M. Samoć and M. G. Humphrey, Coord. Chem. Rev., 2011, 255, 2025-2038.

48 O. Mongin, T. R. Krishna, M. H. V. Werts, A.-M. Caminade, J.-P. Majoral and M. Blanchard-Desce, Chem. Commun., 2006, 915-917.

49 O. Mongin, A. Pla-Quintana, F. Terenziani, D. Drouin, C. L. Droumaguet, A.-M. Caminade, J.-P. Majoral and M. Blanchard-Desce, New J. Chem., 2007, 31, 1354-1367.

50 W. Bartkowiak, R. Zaleśny and J. Leszczynski, Chem. Phys., 2003, 287, 103-112.

51 Y. M. Poronik, V. Hugues, M. Blanchard-Desce and D. T. Gryko, Chem. - Eur. J., 2012, 18, 9258-9266.

52 M. Wielgus, R. Zaleśny, N. A. Murugan, J. Kongsted, H. Ågren, M. Samoć and W. Bartkowiak, ChemPhysChem, 2013, 14, 3731-3739.

53 M. Wielgus, J. Michalska, M. Samoc and W. Bartkowiak, Dyes Pigm., 2015, 113, 426-434.

54 H. Y. Woo, D. Korystov, A. Mikhailovsky, T. Q. Nguyen and G. C. Bazan, J. Am. Chem. Soc., 2005, 127, 13794-13795.

55 Z. A. Dreger, G. Yang, J. O. White, Y. Li and H. G. Drickamer, J. Phys. Chem. B, 1998, 102, 4380-4385.

56 J. Yu, Y. Cui, C. Wu, Y. Yang, Z. Wang, M. O'Keeffe, B. Chen and G. Qian, Angew. Chem., Int. Ed., 2012, 51, 10542-10545. 
57 J. Yu, Y. Cui, H. Xu, Y. Yang, Z. Wang, B. Chen and G. Qian, Nat. Commun., 2013, 4, 2719.

58 K. Kamada, Y. Tanamura, K. Ueno, K. Ohta and H. Misawa, J. Phys. Chem. C, 2007, 111, 11193-11198.

59 Y. Suzuki, Y. Tenma, Y. Nishioka and J. Kawamata, Chem. - Asian J., 2012, 7, 1170-1179.

60 Y. Cui, B. Li, H. He, W. Zhou, B. Chen and G. Qian, Acc. Chem. Res., 2016, 49, 483-493.

61 H. Partridge and R. S. Langhoff, J. Chem. Phys., 1981, 74, 2361-2371.

62 B. Jeziorski, H. J. Monkhorst, K. Szalewicz and J. G. Zabolitzky, J. Chem. Phys., 1984, 81, 368-388.

63 S. Ben-Shlomo and U. Kaldor, J. Chem. Phys., 1988, 89, 956-958.

64 X. Li and J. Paldus, J. Chem. Phys., 2003, 118, 2470-2481.

65 W.-C. Tung, M. Pavanello and L. Adamowicz, J. Chem. Phys., 2011, 134, 064117.

66 B. O. Roos and A. J. Sadlej, J. Chem. Phys., 1982, 76, 5444-5451.

67 M. Cafiero and L. Adamowicz, J. Chem. Phys., 2002, 116, 5557-5564.

68 A. V. Shtoff, M. Rerat and S. I. Gusarov, Eur. Phys. J. D, 2001, 15, 199-208.

69 M. F. Costa and M. C. C. Ribeiro, Quim. Nova, 2006, 29, 1266-1269.

70 W. Cencek, J. Komasa and K. Szalewicz, J. Chem. Phys., 2011, 135, 014301.

71 M. Aymar, J. Deiglmayr and O. Dulieu, Can. J. Phys., 2009, 87, 543-556.

72 J. Kobus, D. Moncrieff and S. Wilson, J. Phys. B: At. Mol. Phys., 2001, 34, 5127-5143.

73 D. M. Bishop and B. Lam, Chem. Phys. Lett., 1985, 120, 69-74.

74 M. Mérawa, D. Bégué and A. Dargelos, J. Phys. Chem. A, 2003, 107, 9628-9633.

75 D. Tunega and J. Noga, Theor. Chem. Acc., 1998, 100, 78-84.

76 D. Theis, Y. G. Khait, S. Pal and M. R. Hoffmann, Chem. Phys. Lett., 2010, 487, 116-121.

$77 \mathrm{Z}$. Hu, J. Autschbach and L. Jensen, J. Chem. Theory Comput., 2016, 12, 1294-1304.

78 L. Jacak, P. Hawrylak and A. Wójs, Quantum Dot, Springer, New York, 1998.

79 J. Karwowski, THEOCHEM, 2005, 727, 1.

80 W. M. McClain and R. A. Harris, Excited States, Academic Press, New York, 1977, vol. 3.

81 P. Norman, P. Cronstrand and J. Ericsson, Chem. Phys., 2002, 285, 207-220.

82 P. R. Monson and W. M. McClain, J. Chem. Phys., 1970, 53, 29-37.

83 P. Cronstrand, Y. Luo and H. Ågren, Chem. Phys. Lett., 2002, 352, 262-269.
$84 \mathrm{~W}$. Bartkowiak and R. Zaleśny, in Non-linear Optical Properties of Matter: From Molecules to Condensed Phases, ed. M. G. Papadopoulos, A. J. Sadlej and J. Leszczyński, Springer, Berlin, 2006, p. 129.

85 Dalton, a molecular electronic structure program, Release Dalton2015.0, 2015, see http://daltonprogram.org.

86 J. Olsen and P. Jørgensen, J. Chem. Phys., 1985, 82, 3235-3264.

87 O. Vahtras, H. Ågren, P. Jørgensen, H. J. A. Jensen, S. B. Padkjaer and T. Helgaker, J. Chem. Phys., 1992, 96, 6120-6125.

88 H. Hettema, H. Jørgen, A. Jensen, P. Jørgensen and J. Olsen, J. Chem. Phys., 1992, 97, 1174-1190.

89 D. Sundholm, J. Olsen and P. Jørgensen, J. Chem. Phys., 1995, 102, 4143-4150.

90 P. O. Widmark, P. A. Malmqvist and B. O. Roos, Theor. Chim. Acta, 1990, 77, 291-306.

91 A. Borgoo, D. J. Tozer, P. Geerlings and F. De Proft, Phys. Chem. Chem. Phys., 2009, 11, 2862.

92 D. M. Bishop and L. M. Cheung, Chem. Phys. Lett., 1979, 66, 467-470.

93 D. M. Bishop and S. A. Solunac, Chem. Phys. Lett., 1985, 122, 567-571.

94 C. E. Dykstra, S.-Y. Liu and D. J. Malik, THEOCHEM, 1986, 135, 357-368.

95 C. E. Dykstra, J. Chem. Educ., 1988, 65, 198.

96 J. D. Augspurger and C. E. Dykstra, J. Chem. Phys., 1988, 88, 3817.

97 S. Huzinaga, E. Miyoshi and M. Sekiya, J. Comput. Chem., 1993, 14, 1440-1445.

98 J. F. Harrison, J. Phys. Chem. A, 2006, 110, 10848-10857.

99 M. A. Buldakov, V. N. Cherepanov, E. V. Koryukina and Y. N. Kalugina, J. Phys. B: At., Mol. Opt. Phys., 2009, 42, 105102.

100 M. Alipour and A. Mohajeri, Chem. Phys., 2011, 387, 5-10.

101 G. Maroulis, Chem. Phys. Lett., 2000, 318, 181-189.

102 G. Maroulis, Chem. Phys. Lett., 2007, 442, 265-269.

103 G. Maroulis, Int. J. Quantum Chem., 2011, 111, 807-818.

104 G. Maroulis, Theor. Chem. Acc., 2011, 129, 437-445.

105 H. Habli, L. Mejrissi, H. Ghalla, S. J. Yaghmour, B. Oujia and F. X. Gadéa, Mol. Phys., 2016, 114, 1568-1582.

106 E. Zicler, M.-C. Bacchus-Montabonel, F. Pauzat, P. Chaquin and Y. Ellinger, J. Chem. Phys., 2016, 144, 111103.

107 O. Loboda, F. Ingrosso, M. F. Ruiz-López, H. Reis and C. Millot, J. Comput. Chem., 2016, 37, 2125-2132.

108 J. M. H. Lo, M. Klobukowski, D. Bielińska-Waż, E. W. S. Schreiner and G. H. F. Diercksen, J. Phys. B: At., Mol. Opt. Phys., 2006, 39, 2385-2402.

109 O. Quinet and B. Champagne, J. Chem. Phys., 2001, 115, 6293. 